\title{
International normalized ratio on admission predicts the 90-day mortality of critically ill patients undergoing endarterectomy
}

\author{
LANG-PING TAN ${ }^{1 *}$, YI-BIAO YE ${ }^{2 *}$, YUE ZHU ${ }^{1}$, ZHI-LONG GU ${ }^{3}$, QIN-GUI CHEN ${ }^{4}$ and MIAO-YUN LONG ${ }^{1}$ \\ Departments of ${ }^{1}$ Vascular Surgery and ${ }^{2}$ Hepatobiliary Surgery, Sun Yat-Sen Memorial Hospital of Sun Yat-Sen University, \\ Guangzhou, Guangdong 510000; ${ }^{3}$ Intensive Care Unit, First Affiliated Hospital of Jinzhou Medical University, \\ Jinzhou, Liaoning 121001; ${ }^{4}$ Medical Intensive Care Unit, First Affiliated Hospital of Sun Yat-Sen University, \\ Guangzhou, Guangdong 510080, P.R. China
}

Received April 24, 2018; Accepted October 3, 2018

DOI: $10.3892 /$ etm.2018.6935

\begin{abstract}
The association of the international normalized ratio (INR) with the long-term clinical outcome of patients who undergo endarterectomy has not yet been studied. The present study therefore primarily aimed to evaluate the association of INR on admission with the 90-day mortality of critically ill patients who underwent endarterectomy during hospitalization. The Medical Information Mart for Intensive Care III database was queried for patients undergoing endarterectomy. The 90-day mortality of patients was selected as a primary endpoint. Receiver-operating characteristic (ROC) curves were plotted to present the accuracy of predictions. Kaplan-Meier curves and multivariate Cox regression analysis were performed to analyse associations. Propensity score matching (PSM) was also conducted to reduce confounding bias. A total of 230 patients were included, with 36 90-day non-survivors. Patients with a high INR $(\geq 1.5)$ on admission exhibited a higher 90-day mortality than those with a low INR $(<1.5 ; 29.09$ vs. $11.43 \%$; $\mathrm{P}=0.003)$. The ROC area under the curve value was 0.687 [95\% confidence interval (CI), 0.571-0.780]. Kaplan-Meier plots identified divergence in survival between patients with different INR levels (log-rank test, $\mathrm{P}=0.0013$ ). The results of the multivariate Cox regression analysis indicated that a high INR level was significantly associated with 90-day mortality (hazard ratio, 2.19; $95 \% \mathrm{CI}, 1.08-4.45 ; \mathrm{P}=0.0305)$. Analysis of the PSM
\end{abstract}

Correspondence to: Dr Miao-Yun Long, Department of Vascular Surgery, Sun Yat-Sen Memorial Hospital of Sun Yat-Sen University, 33 Yingfeng Road, Guangzhou, Guangdong 510000, P.R. China

E-mail: 13427584517@163.com

*Contributed equally

Abbreviations: CI, confidence interval; INR, international normalized ratio; SAPS II, simplified acute physiology score II; PSM, propensity score matching; IQR, interquartile range; ROC, receiver operating characteristic; $\mathrm{OR}$, odds ratio

Key words: international normalized ratio, intensive care unit, mortality, prognosis, endarterectomy cohort presented similar results. In conclusion, the INR levels of critically ill patients who undergo endarterectomy may be used to stratify their risk of 90-day mortality.

\section{Introduction}

Endarterectomy, often performed on the carotid, femoral, aortic and pulmonary arteries, is a surgical procedure that removes part of the arterial inner lining along with an adherent atherosclerotic plaque, which aims to recanalize the occluded vessel and restore vascular supply to the tissue $(1,2)$. As one of the most frequently performed peripheral arterial surgeries, it has been demonstrated that the 30-day post-carotid endarterectomy mortality was $1.40 \%$ [95\% confidence interval (CI) 1.34-1.47] from 2001 to 2002 and $1.17 \%$ (CI, 1.10-1.24) from 2007 to 2008 (3). A recent study has revealed a 3.4\% 30-day mortality following isolated common femoral endarterectomy (4). However, the mortality rate of emergency carotid revascularization has risen to $\sim 10 \%$ in Germany under routine conditions (5). Considering the severity of patient illness in intensive care units, it is not uncommon for there to be a poorer outcome for those who undergo endarterectomy, although associated epidemiological data are limited. As the clinical outcome prediction of critically ill patients may contribute to individual patient management (6), it is necessary to determine useful predictors for such patients. The international normalized ratio (INR) is a routine coagulation test, which has been determined to be a predictor of several clinical conditions, including mortality in patients with trauma $(7,8)$, risk stratification for patients with acute ischemic stroke that are treated with warfarin (9) and mortality in patients with sepsis (10). However, to the best of our knowledge, it remains unknown whether the INR on admission could serve as a reliable predictor of long-term outcome for critically ill patients who undergo endarterectomy following admission. Therefore, the current study extracted data from a publicly accessible database of critical care medicine to investigate the prognostic predictive value of INR.

\section{Patients and methods}

Database. The Medical Information Mart for Intensive Care III database is a large, publicly available database consisting of 
de-identified data for patients that were hospitalized in the intensive care unit at Beth Israel Deaconess Medical Centre (Boston, MA, USA) between 2001 and 2012 (11). Access to this database was approved by the institutional review board of Beth Israel Deaconess Medical Centre and Massachusetts Institute of Technology Affiliates (Boston, MA, USA). No informed consent was required.

Patients. Patients with endarterectomy records during hospitalization were identified by International Classification of Diseases, Ninth Revision procedure codes (381.0-381.6, 381.8) and included. Patients that were $\geq 18$ years of age who remained in hospital for $\geq 1$ day were included into the present study. Patients were excluded from the current study if their INR records were obtained $>24$ h following hospitalization (Fig. 1). A total of 230 patients were included into the present study. The median age of the patients was 74.61 years (IQR 67.33-81.34 years) and 121 of the 230 cases $(52.61 \%)$ were male.

Variables. Structured Query Language was applied to extract data from the database primarily using codes obtained from the Medical Information Mart for Intensive Care Code Repository (github.com/MIT-LCP/mimic-code) (12). The following variables were extracted or calculated: Age, sex, 90-day mortality, hospital mortality, length of hospital stay, INR, simplified acute physiology score II (SAPS II) (13), Elixhauser comorbidities (14), the Elixhauser Comorbidity Index (SID30) (15), primary diagnosis and type of endarterectomy. Missing components for the calculation of SAPS II were treated as normal (usually 0) (12).

Outcomes. The 90-day mortality following hospital admission was selected as the primary outcome prior to data extraction and analysis. Hospital mortality was considered to be the secondary outcome. The length of hospitalization was calculated only for statistical description.

Propensity score matching (PSM). Patients were categorized as survivors or non-survivors according to their 90-day survival status following hospital admission. The propensity score for each patient was calculated to estimate the probability of mortality in the first 90 days following hospital admission using multivariable logistic regression models that adjusted the following covariates: Age, sex, SAPS II, SID30, type of endarterectomy and comorbidities, which included the following: Congestive heart failure, cardiac arrhythmia, valvular disease, pulmonary circulation disorder, peripheral vascular disorder, hypertension, paralysis, other neurological diseases, chronic pulmonary disease, uncomplicated diabetes, complicated diabetes, hypothyroidism, renal failure, liver disease, lymphoma, metastatic cancer, solid tumor, rheumatoid arthritis, coagulopathy, obesity, weight loss, fluid and electrolyte disorders, blood loss anemia, deficiency anemia, alcohol abuse, drug abuse, psychosis and depression. Matching was performed using a 1:1 matching protocol without replacement (greedy-matching algorithm), with a caliper width equal to 0.05 of the standard deviation of the logit of the propensity score.

Statistical analysis. Data are presented as the median and interquartile range (IQR) for continuous variables and as numbers

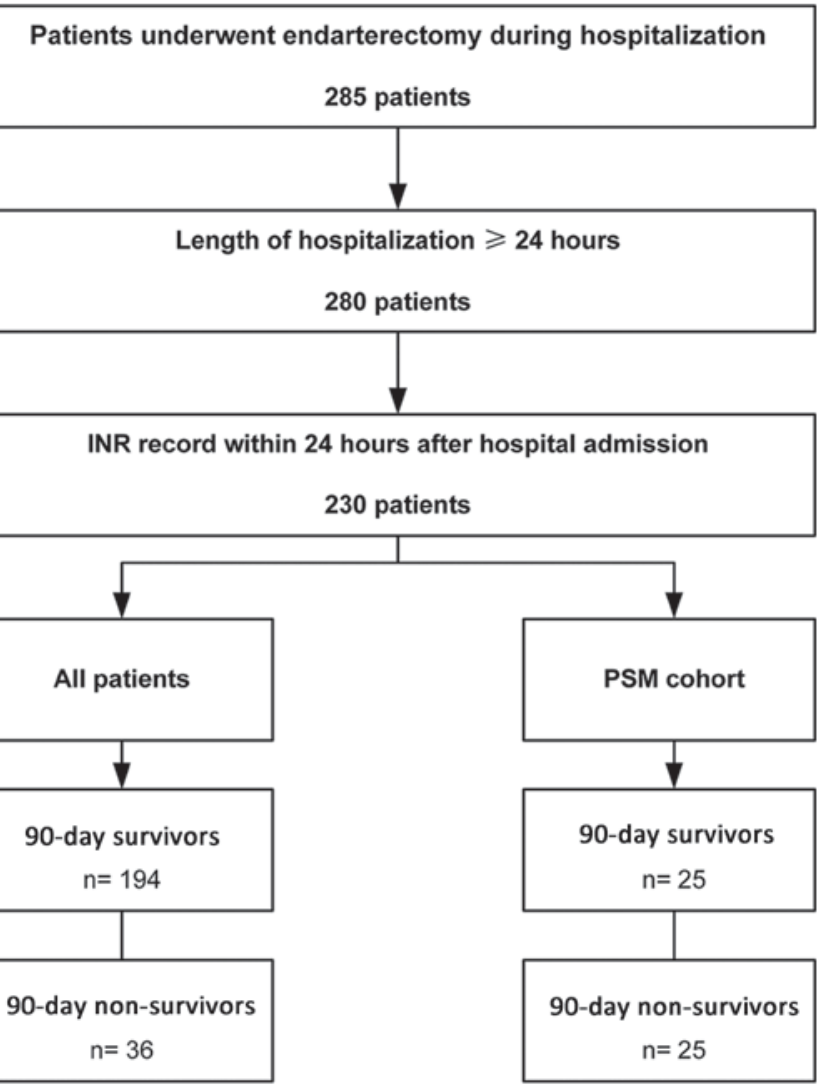

Figure 1. Flow chart of the current study. Patients who underwent endarterectomy during hospitalization were screened. Those with a length of hospitalization over $24 \mathrm{~h}$ with INR records available were included. INR, international normalized ratio; PSM, propensity score matching.

and percentages for categorical variables. The comparisons of continuous and categorical variables were performed using Kruskal Wallis and $\mathrm{X}^{2}$ (or Fisher's exact) tests, respectively. Receiver operating characteristic (ROC) curves were performed to evaluate the prognostic predictive value of INR for 90-day mortality and hospital mortality. The best cut-off value was determined using Youden's Index and patients were subsequently grouped as high and low INR according to this cut-off value. Kaplan-Meier curves were plotted and log-rank tests were used to compare survival between the two groups. Variables associated with 90-day mortality were evaluated using univariate Cox regression analysis and those with a value of $\mathrm{P}<0.1$ were included in the multivariable Cox regression model. Age was not included in the multivariable regression analysis since it was factored into SAPS II. The multivariable Cox regression model was performed to evaluate the association of INR with 90-day mortality and the multivariable logistic regression model was used to evaluate the association of INR with hospital mortality. $\mathrm{P}<0.05$ was considered to indicate a statistically significant difference. Empower(R) (www.empowerstats.com; $\mathrm{X} \& Y$ Solutions, Inc., Boston MA) and R software (version 3.4.3; www.r-project.org) were used for statistical analyses.

\section{Results}

Characteristics of the patients. The median admission INR was 1.30 (IQR, 1.10-1.40) with a median SAPS II score of 36 (IQR, 29-43; Table I). A total of $42.61 \%$ patients underwent 
Table I. Characteristics of all patients.

\begin{tabular}{|c|c|c|c|c|}
\hline Variable & All patients $(n=230)$ & INR < $1.5(n=175)$ & INR $\geq 1.5(\mathrm{n}=55)$ & P-value \\
\hline Age (years) & $74.61(67.33-81.34)$ & $73.63(66.68-81.26)$ & $76.53(68.68-81.46)$ & 0.350 \\
\hline Male & $121(52.61 \%)$ & $91(52.00 \%)$ & $30(54.55 \%)$ & 0.742 \\
\hline 90-day mortality & $36(15.65 \%)$ & $20(11.43 \%)$ & $16(29.09 \%)$ & 0.003 \\
\hline Hospital mortality & $22(9.57 \%)$ & $11(6.29 \%)$ & $11(20.00 \%)$ & 0.006 \\
\hline Median length of hospital stay (days) & $10.89(6.94-17.92)$ & $10.53(6.57-15.79)$ & $13.39(8.44-24.71)$ & 0.013 \\
\hline INR on admission & $1.30(1.10-1.40)$ & $1.20(1.10-1.30)$ & $2.00(1.60-2.50)$ & $<0.001$ \\
\hline SAPS II on admission & $36(29-43)$ & $35(29-42)$ & $39(31.5-46.25)$ & 0.132 \\
\hline SID30 & $9.00(3.00-19.00)$ & $7.00(2.00-15.00)$ & $16.00(4.00-24.50)$ & 0.002 \\
\hline
\end{tabular}

Data are expressed as median (interquartile range) or $\mathrm{n}(\%)$ unless otherwise stated. Kruskal Wallis and $\mathrm{X}^{2}$ (or Fisher's exact) tests were used to analyse continuous and categorical variables, respectively. INR, international normalized ratio; SAPS II, simplified acute physiology score II; SID30, Elixhauser comorbidity index.

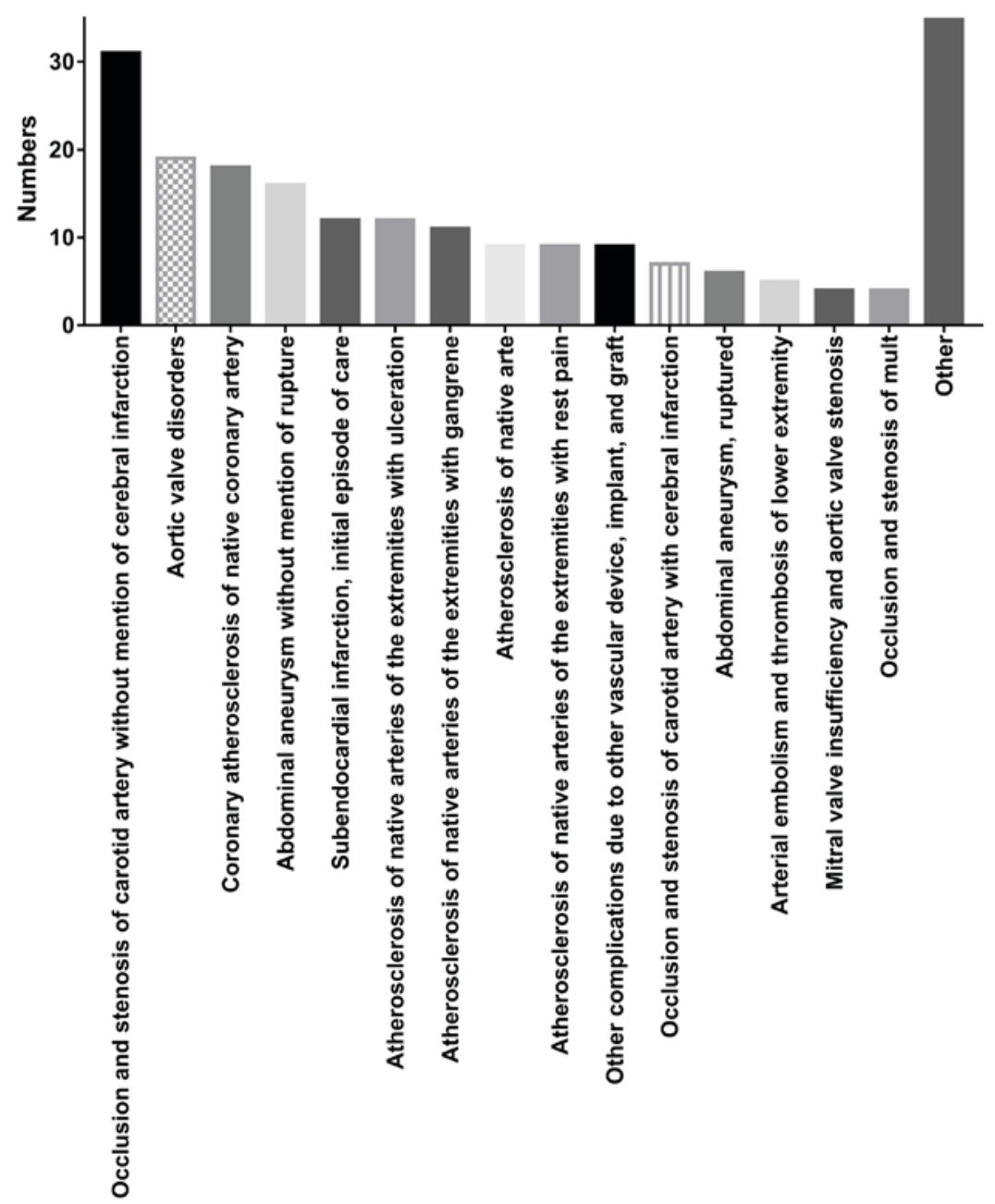

Figure 2. Primary diagnoses of the patients included in the present study. Occlusion and stenosis of the carotid artery without mention of cerebral infarction made up the largest proportion of diagnoses.

lower limb artery endarterectomy, 31.74\% underwent carotid endarterectomy, $22.61 \%$ underwent aorta endarterectomy and 9.13\% underwent abdominal artery endarterectomy (Table II). The five most common comorbidities were peripheral vascular disorder $(53.04 \%)$, chronic pulmonary disease $(31.30 \%)$, uncomplicated diabetes (30.43\%), fluid and electrolyte disorders
(22.61\%), and renal failure (21.74\%). The 90-day mortality was $15.65 \%$ with 36 non-survivors and 194 survivors (Table I). The median length of hospital stay was 10.89 days (IQR, 6.94-17.92). Primary patient diagnoses are presented in Fig. 2. The five most common primary diagnoses were occlusion and stenosis of carotid artery without cerebral infarction, aortic valve disorders, 
Table II. Types of endarterectomy and comorbidities of all patients.

\begin{tabular}{|c|c|c|c|c|}
\hline Variable & All patients $(\mathrm{n}=230)(\%)$ & INR $<1.5(\mathrm{n}=175)(\%)$ & INR $\geq 1.5(\mathrm{n}=55)(\%)$ & P-value \\
\hline \multicolumn{5}{|l|}{ Endarterectomy } \\
\hline Other vessels of head and neck & $73(31.74)$ & $61(34.86)$ & $12(21.82)$ & 0.070 \\
\hline Aorta & $52(22.61)$ & $36(20.57)$ & $16(29.09)$ & 0.188 \\
\hline Other thoracic vessels & $1(0.43)$ & $1(0.57)$ & $0(0.00)$ & 1.000 \\
\hline Abdominal arteries & $21(9.13)$ & $15(8.57)$ & $6(10.91)$ & 0.596 \\
\hline Lower limb arteries & $98(42.61)$ & $75(42.86)$ & $23(41.82)$ & 0.892 \\
\hline \multicolumn{5}{|l|}{ Comorbidities } \\
\hline Congestive heart failure & $21(9.13)$ & $14(8.00)$ & $7(12.73)$ & 0.291 \\
\hline Cardiac arrhythmias & $31(13.48)$ & $20(11.43)$ & $11(20.00)$ & 0.116 \\
\hline Valvular disease & $9(3.91)$ & $6(3.43)$ & $3(5.45)$ & 0.450 \\
\hline Pulmonary circulation disorder & $1(0.43)$ & $0(0.00)$ & $1(1.82)$ & 0.239 \\
\hline Peripheral vascular disorder & $122(53.04)$ & $87(49.71)$ & $35(63.64)$ & 0.071 \\
\hline Hypertension & $40(17.39)$ & $29(16.57)$ & $11(20.00)$ & 0.546 \\
\hline Paralysis & $6(2.61)$ & $3(1.71)$ & $3(5.45)$ & 0.150 \\
\hline Other neurological disease & $10(4.35)$ & $6(3.43)$ & $4(7.27)$ & 0.256 \\
\hline Chronic pulmonary disease & $72(31.30)$ & $57(32.57)$ & $15(27.27)$ & 0.460 \\
\hline Uncomplicated diabetes & $70(30.43)$ & $54(30.86)$ & $16(29.09)$ & 0.804 \\
\hline Complicated diabetes & $30(13.04)$ & $22(12.57)$ & $8(14.55)$ & 0.654 \\
\hline Hypothyroidism & $24(10.43)$ & $21(12.00)$ & $3(5.45)$ & 0.211 \\
\hline Renal failure & $50(21.74)$ & $36(20.57)$ & $14(25.45)$ & 0.444 \\
\hline Liver disease & $8(3.48)$ & $4(2.29)$ & $4(7.27)$ & 0.096 \\
\hline Lymphoma & $2(0.87)$ & $2(1.14)$ & $0(0.00)$ & 1.000 \\
\hline Metastatic cancer & $3(1.30)$ & $2(1.14)$ & $1(1.82)$ & 0.561 \\
\hline Solid tumor & $2(0.87)$ & $2(1.14)$ & $0(0.00)$ & 1.000 \\
\hline Rheumatoid arthritis & $7(3.04)$ & $5(2.86)$ & $2(3.64)$ & 0.673 \\
\hline Coagulopathy & $34(14.78)$ & $20(11.43)$ & $14(25.45)$ & 0.016 \\
\hline Obesity & $12(5.22)$ & $11(6.29)$ & $1(1.82)$ & 0.302 \\
\hline Weight loss & $9(3.91)$ & $6(3.43)$ & $3(5.45)$ & 0.450 \\
\hline Fluid and electrolyte disorders & $52(22.61)$ & $34(19.43)$ & $18(32.73)$ & 0.040 \\
\hline Blood loss anemia & $3(1.30)$ & $1(0.57)$ & $2(3.64)$ & 0.143 \\
\hline Deficiency anemia & $37(16.09)$ & $26(14.86)$ & $11(20.00)$ & 0.401 \\
\hline Alcohol abuse & $6(2.61)$ & $6(3.43)$ & $0(0.00)$ & 0.340 \\
\hline Drug abuse & $1(0.43)$ & $1(0.57)$ & $0(0.00)$ & 1.000 \\
\hline Psychoses & $2(0.87)$ & $2(1.14)$ & $0(0.00)$ & 1.000 \\
\hline Depression & $7(3.04)$ & $4(2.29)$ & $3(5.45)$ & 0.362 \\
\hline
\end{tabular}

Data are expressed as median (interquartile range) or $\mathrm{n}(\%)$ unless otherwise stated. Kruskal Wallis and $\mathrm{X}^{2}$ (or Fisher's exact) tests were used to analyse continuous and categorical variables, respectively. INR, International Normalized Ratio.

coronary atherosclerosis of native coronary artery, abdominal aneurysm without rupture and subendocardial infarction. A total of 25 non-survivors were successfully matched with one control. The characteristics of the PSM cohort are presented in Tables III and IV. Patients with a high INR on admission still exhibited a higher 90-day mortality than those with a low INR (86.67 vs. $34.29 \%$; $\mathrm{P}=0.001$ ). No statistically significant difference was identified between survivors and non-survivors in age, sex, SAPS II on admission and SID30 (data not shown).

ROC curve analysis. Area under the ROC curve values of admission INR for the discrimination of 90-day survivors and non-survivors were 0.687 (95\% CI, 0.571-0.780) and 0.794 (95\% CI, 0.569-0.863) in all patients and the PSM cohort, respectively (Fig. 3). The best cut-off value of INR was 1.55, as determined using Youden's index. Therefore, patients were further categorized as high INR (INR $\geq 1.5$ ) and low INR (INR <1.5).

Clinical outcomes of patients with different INR levels on admission. As presented in Tables I and III, patients with a high INR exhibited a significantly higher 90-day mortality compared with patients with a low INR. Kaplan-Meier curves also demonstrated similar results (Fig. 4). Additionally, 
Table III. Characteristics of the PSM cohort.

\begin{tabular}{|c|c|c|c|c|}
\hline Variable & All patients $(\mathrm{n}=50)$ & INR $<1.5(\mathrm{n}=35)$ & INR $\geq 1.5(\mathrm{n}=15)$ & P-value \\
\hline Age (years) & $77.81(69.94-82.76)$ & $76.68(69.31-82.41)$ & $80.84(76.61-82.89)$ & 0.208 \\
\hline Male & $35(70.00 \%)$ & $26(74.29 \%)$ & $9(60.00 \%)$ & 0.333 \\
\hline 90-day mortality & $25(50.00 \%)$ & $12(34.29 \%)$ & $13(86.67 \%)$ & 0.001 \\
\hline Hospital mortality & $16(32.00 \%)$ & $6(17.14 \%)$ & $10(66.67 \%)$ & 0.002 \\
\hline Length of hospital stay (days) & $13.04(9.71-24.62)$ & $12.90(9.98-23.01)$ & $19.89(9.27-29.73)$ & 0.619 \\
\hline INR on admission & $1.30(1.20-1.75)$ & $1.20(1.10-1.30)$ & $2.30(1.80-3.45)$ & $<0.001$ \\
\hline SAPS II on admission & $41.5(33-55)$ & $42(34-55)$ & $39(33-48)$ & 0.596 \\
\hline SID30 & $12.50(3.25-19.75)$ & $12.00(3.00-16.50)$ & $18.00(6.50-24.50)$ & 0.155 \\
\hline
\end{tabular}

Data are expressed as median (interquartile range) or $\mathrm{n}(\%)$ unless otherwise stated. Kruskal Wallis and $\mathrm{X}^{2}$ (or Fisher's exact) tests were used to analyse continuous and categorical variables, respectively. PSM, propensity score matching; INR, international normalized ratio; SAPS II, simplified acute physiology score II; SID30, Elixhauser comorbidity index.
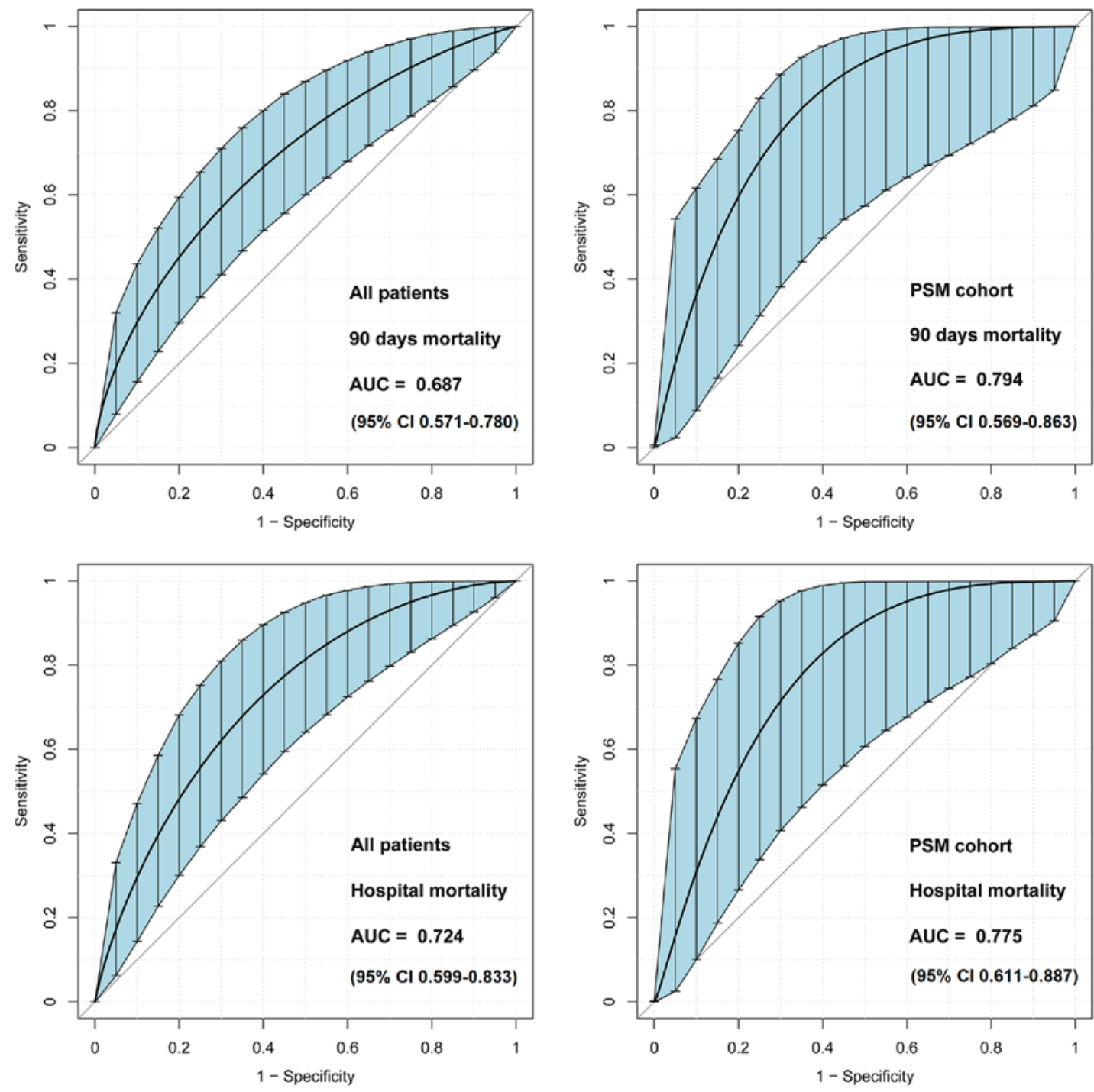

Figure 3. ROC curves of admission INR for the prediction of patient clinical outcomes. INR on admission exhibited fair discriminatory power for 90-day mortality and hospital mortality. The bootstrap ROC is used to determine a confidence interval around an estimated AUC. ROC curves, receiver operating characteristic curves; INR, international normalized ratio; AUC, area under the curve; PSM, propensity score matching.

patients with a high INR exhibited a higher hospital mortality than those with a low INR; however, there was no significant difference of length of hospital stay according to the PSM cohort analyses. 
Table IV. Types of endarterectomy and comorbidities of the PSM cohort.

\begin{tabular}{lcccc}
\hline Variable & All patients $(\mathrm{n}=50)(\%)$ & INR $<1.5(\mathrm{n}=35)(\%)$ & INR $\geq 1.5(\mathrm{n}=15)(\%)$ & P-value \\
\hline Endarterectomy & & & & \\
Other vessels of head and neck & $9(18.00)$ & $8(22.86)$ & $1(6.67)$ & 0.247 \\
Aorta & $8(16.00)$ & $5(14.29)$ & $3(20.00)$ & 0.683 \\
Abdominal arteries & $3(6.00)$ & $1(2.86)$ & $2(13.33)$ & 0.211 \\
Lower limb arteries & $31(62.00)$ & $22(62.86)$ & $9(60.00)$ & 1.000 \\
Comorbidities & & & & \\
Congestive heart failure & $3(6.00)$ & $3(8.57)$ & $0(0.00)$ & 0.545 \\
Cardiac arrhythmias & $5(10.00)$ & $4(11.43)$ & $1(6.67)$ & 1.000 \\
Valvular disease & $2(4.00)$ & $1(2.86)$ & $1(6.67)$ & 0.514 \\
Peripheral vascular disorder & $30(60.00)$ & $19(54.29)$ & $11(73.33)$ & 0.345 \\
Hypertension & $14(28.00)$ & $10(28.57)$ & $4(26.67)$ & 1.000 \\
Paralysis & $1(2.00)$ & $1(2.86)$ & $0(0.00)$ & 1.000 \\
Other neurological disease & $2(4.00)$ & $1(2.86)$ & $1(6.67)$ & 0.514 \\
Chronic pulmonary disease & $14(28.00)$ & $9(25.71)$ & $5(33.33)$ & 0.733 \\
Uncomplicated diabetes & $4(8.00)$ & $1(2.86)$ & $3(20.00)$ & 0.075 \\
Complicated diabetes & $4(8.00)$ & $2(5.71)$ & $2(13.33)$ & 0.574 \\
Hypothyroidism & $2(4.00)$ & $1(2.86)$ & $1(6.67)$ & 0.514 \\
Renal failure & $15(30.00)$ & $11(31.43)$ & $4(26.67)$ & 1.000 \\
Liver disease & $2(4.00)$ & $0(0.00)$ & $2(13.33)$ & 0.086 \\
Solid tumor & $1(2.00)$ & $1(2.86)$ & $0(0.00)$ & 1.000 \\
Rheumatoid arthritis & $1(2.00)$ & $0(0.00)$ & $1(6.67)$ & 0.300 \\
Coagulopathy & $11(22.00)$ & $6(17.14)$ & $5(33.33)$ & 0.269 \\
Obesity & $3(6.00)$ & $3(8.57)$ & $0(0.00)$ & 0.545 \\
Weight loss & $3(6.00)$ & $1(2.86)$ & $2(13.33)$ & 0.211 \\
Fluid and electrolyte disorders & $16(32.00)$ & $9(25.71)$ & $7(46.67)$ & 0.191 \\
Deficiency anemia & $5(10.00)$ & $3(8.57)$ & $2(13.33)$ & 0.629 \\
Alcohol abuse & $1(2.00)$ & $1(2.86)$ & $0(0.00)$ & 1.000 \\
Depression & $1(2.00)$ & $0(0.00)$ & $1(6.67)$ & 0.300 \\
\hline Dian & & & \\
\hline
\end{tabular}

Data are expressed as median (interquartile range) or n (\%) unless otherwise stated. Kruskal Wallis and $\mathrm{X}^{2}$ (or Fisher's exact) tests were used to analyse continuous and categorical variables, respectively. PSM, propensity score matching; INR, international normalized ratio.

Association of INR levels on admission and clinical outcomes. The results of the univariate Cox regression analysis of all patients and the PSM cohort are presented in Tables V and VI, respectively. SAPS II, INR and SID30 were significantly associated with the 90-day mortality of all patients. Multivariable regression analysis (Table VII) indicated that a high INR was an independent risk factor of 90-day mortality (hazard ratio, 2.19; 95\% CI, 1.08-4.45) and hospital mortality (odds ratio $2.59,95 \%$ CI 1.01-6.66). The results of the PSM cohort regression analysis are also presented in Table VII, which indicated a significant association of a high INR with 90-day mortality (hazard ratio, 4.18; 95\% CI, 1.86-9.38) and hospital mortality (odds ratio 9.67; $95 \%$ CI 2.41-38.71).

\section{Discussion}

The present study examined the association of INR on hospital admission and clinical outcomes in critically ill patients who underwent endarterectomy during hospitalization. To the best of our knowledge, the current study is the first to focus on the association of INR and the outcome of patients undergoing endarterectomy. The results indicated that a high INR on admission predicted a poorer long-term patient outcome.

A high INR has been determined to be a marker for bleeding risk (16). For example, an INR $>4$ is associated with an increased risk of bleeding (17) and the risk of intracranial haemorrhage increases 2 -fold for every 1 unit rise in INR (18). Therefore, it is reasonable to assume that patients with a higher INR may have poorer clinical outcomes, regardless of the conditions that they suffer. However, Hansen et al (19) did not identify any clinically relevant difference in the INR values of patients who did or did not develop pulmonary embolism. Furthermore, Adike et al (20) demonstrated that INR was not a significant factor in the prediction of bleeding risk in patients with cirrhosis following endoscopic retrograde cholangiopancreatography. Therefore, it remains unknown whether INR levels are associated with the outcome of patients undergoing endarterectomy. As aforementioned, critically ill patients undergoing endarterectomy exhibited a 
Table V. Univariate Cox regression analysis of the 90-day mortality of all patients.

\begin{tabular}{lccc}
\hline Variable & HR & $95 \% \mathrm{CI}$ & P-value \\
\hline $\begin{array}{l}\text { Age (years) } \\
\text { Sex }\end{array}$ & 1.00 & $1.00-1.01$ & 0.0498 \\
$\quad$ Male & 1.00 & & \\
Female & 0.69 & $0.35-1.35$ & 0.2748 \\
SAPS II & 1.03 & $1.01-1.05$ & 0.0076 \\
INR & & & \\
$<1.5$ & 1.00 & & 0.0021 \\
$\geq 1.5$ & 2.81 & $1.46-5.43$ & 0.0029 \\
SID30 & 1.04 & $1.01-1.07$ & \\
\hline
\end{tabular}

HR, hazard ratio; CI, confidence interval; SAPS II, simplified acute physiology score II; INR, international normalized ratio; SID30, exlixhauser comorbidity index.

Table VI. Univariate Cox regression analysis of the 90-day mortality of the PSM.

\begin{tabular}{lccc}
\hline Variable & HR & $95 \%$ CI & P-value \\
\hline Age (years) & 1.00 & $0.99-1.01$ & 0.4658 \\
Sex & & & \\
$\quad$ Male & 1.00 & & \\
Female & 1.22 & $0.53-2.84$ & 0.6389 \\
SAPS II & 0.99 & $0.97-1.02$ & 0.6438 \\
INR & & & \\
$<1.5$ & 1.00 & & \\
$\geq 1.5$ & 4.18 & $1.86-9.38$ & 0.0005 \\
SID30 & 0.99 & $0.96-1.03$ & 0.7377 \\
\hline
\end{tabular}

PSM, propensity score matching; HR, hazard ratio; CI, confidence interval; SAPS II, simplified acute physiology score II; INR, international normalized ratio; SID30, elixhauser comorbidity index.

markedly higher mortality rate (5). Therefore, it is necessary to determine whether the association exists in critically ill patients. The results of the current study indicated that the risk of 90-mortality increased 1.19 -fold for critically ill patients undergoing endarterectomy with a high INR, compared with patients with a low INR, which might be helpful when deciding whether to perform endarterectomy on critically ill patients.

Several limitations of the present study need to be discussed. Although the focus of the current study was on critically ill patients, such individuals are difficult to define. The patients involved in the current study came from the critical care medicine database and had mostly been admitted to the intensive care unit during their hospitalization. The primary diagnosis of the study populations varied and this was not adjusted in the analysis due to the limited sample size. A further limitation is that INR fluctuations are common due to drug interactions, changes in dietary vitamin $\mathrm{K}$ intake or
Table VII. Associations of INR with 90-day mortality and hospital mortality.

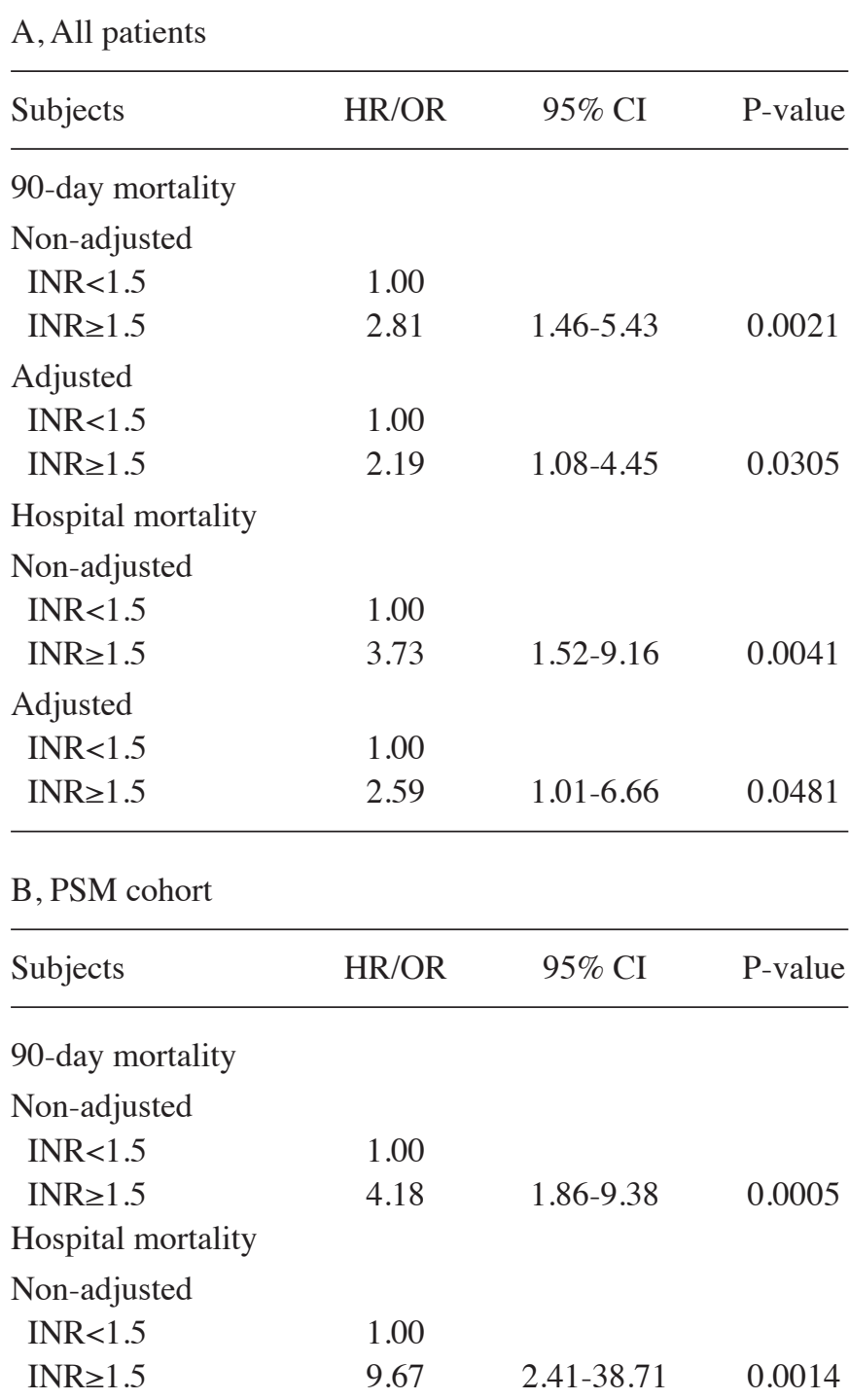

Association of INR with 90-day mortality was analysed using Cox regression models and the association of INR with hospital mortality was analysed using logistic regression models. An adjusted model was used for SAPS II and Elixhauser Comorbidity Index SID30. INR, international normalized ratio; HR, hazard ratio; OR, odds ratio; CI, confidence interval; PSM, propensity score matching; SAPS II, simplified acute physiology score II.

other reasons $(21,22)$, which may affect INR levels on admission. However, this information was difficult to obtain from an electronic database retrospectively. Additionally, although a high INR usually serves as a marker for increased bleeding risk, numerous patients in the current study with prolonged INR do not bleed (data not shown) and the cause of death was not specified due to limited information. Therefore, despite the results of the present study, it remains unknown whether a high INR increases the risk of mortality by increasing bleeding risk.

Other limitations of the present study should be discussed. Although PSM was performed to minimize selection bias, and the analysis of all patients and the PSM cohort presented consistent results, there remained potential unobserved confounding 

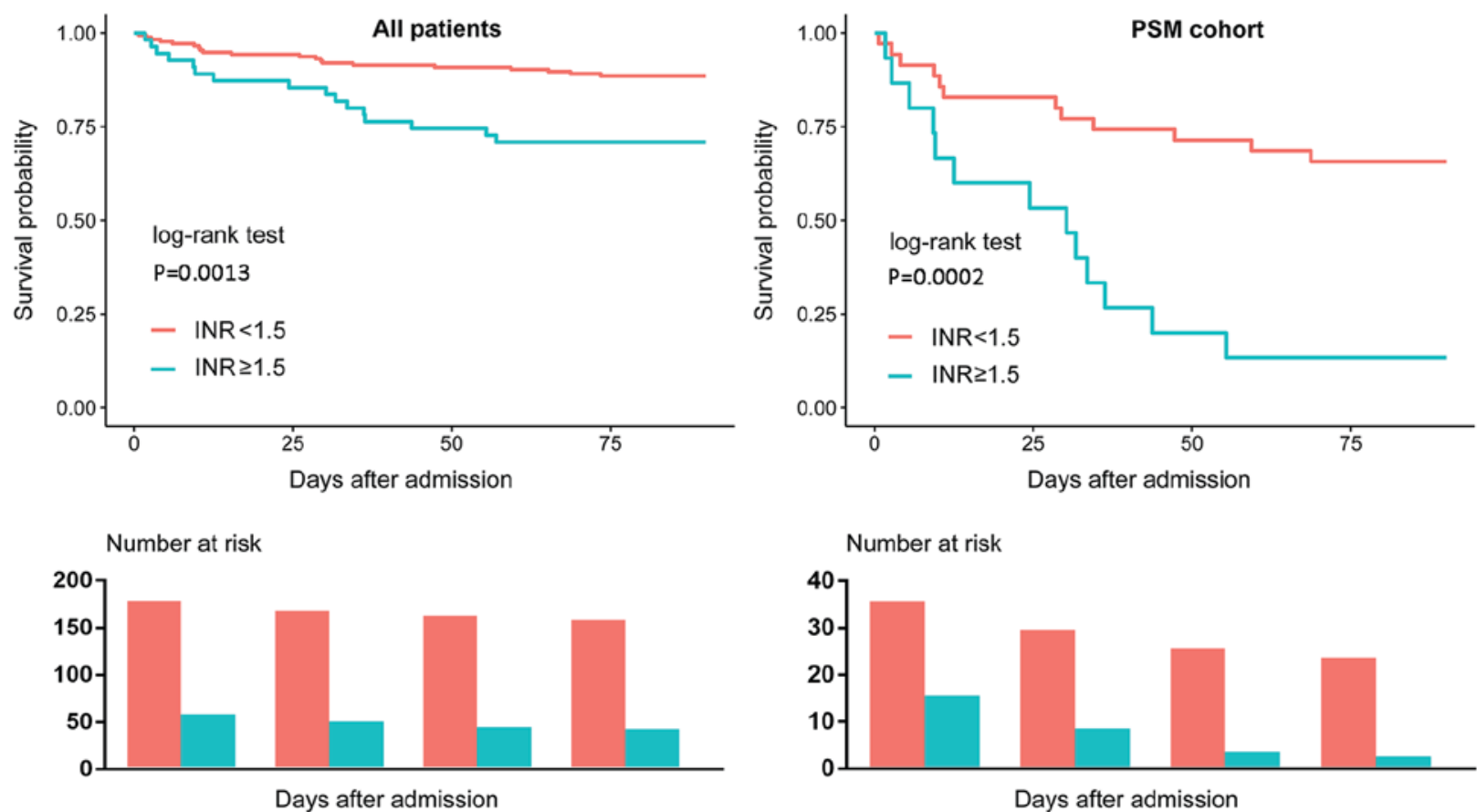

Figure 4. Kaplan-Meier Survival Curve. Patients with INR $\geq 1.5$ exhibited poorer survival compared with patients with an INR $<1.5$. PSM, propensity score matching.

factors which were unadjusted. For example, Mlejnsky et al (23) identified that interleukin-6 (IL-6) may increase in patients following pulmonary endarterectomy; however, whether it was associated with clinical outcome remained unknown following the results of the current study, as IL-6 was not available from the retrospective database. In addition, the sample size of the PSM cohort was small, resulting in a broader confidence interval of the effect. Given the observational nature of the current study, it is not possible to conclude that the association between admission INR and 90-day mortality reflects cause and effect, so it remains unknown whether decreasing the INR levels of critically ill patients could reduce the risk of mortality following endarterectomy. The present study also included data from only one centre, which may limit the external applicability of the results.

In conclusion, an admission INR of $\geq 1.5$ strongly predicts the 90-day mortality of critically ill patients undergoing endarterectomy. These patients may therefore require early aggressive interventions and monitoring, with the aim of improving patient outcome. However, further study is required to validate this.

\section{Acknowledgements}

Not applicable.

\section{Funding}

No funding was received.

\section{Availability of data and materials}

The datasets generated and/or analysed during the current study are available in the PhysioBank repository (mimic. physionet.org).

\section{Authors' contributions}

M-YL designed the current study. M-YL, L-PT, and Y-BY performed data extraction and analysis. YZ, Z-LG, and Q-GC also conducted data analysis. All authors approved the final version of the manuscript.

\section{Ethics approval and consent to participate}

The access of the database was approved by the institutional review boards of both Beth Israel Deaconess Medical Centre and Massachusetts Institute of Technology Affiliates. No informed consent was required because data are anonymized.

\section{Patient consent for publication}

Not applicable since the study did not include any identifying information.

\section{Competing interests}

The authors declare that they have no competing interests.

\section{References}

1. Thompson JE: The evolution of surgery for the treatment and prevention of stroke. The Willis Lecture. Stroke 27: 1427-1434, 1996.

2. Sarkar R and Messina LM: Endarterectomy of the abdominal aorta and its branches. In: Vascular surgery. Edited by Lumley JSP, Hoballah JJ. Berlin, Heidelberg: Springer Berlin Heidelberg; 209-230, 2009.

3. Kumamaru H, Jalbert JJ, Nguyen LL, Gerhard-Herman MD, Williams LA, Chen CY, Seeger JD, Liu J, Franklin JM and Setoguchi S: Surgeon case volume and 30-day mortality after carotid endarterectomy among contemporary medicare beneficiaries: Before and after national coverage determination for carotid artery stenting. Stroke 46: 1288-1294, 2015. 
4. Nguyen BN, Amdur RL, Abugideiri M, Rahbar R, Neville RF and Sidawy AN: Postoperative complications after common femoral endarterectomy. J Vasc Surg 61: 1489-1494, 2015.

5. Knappich C, Kuehnl A, Tsantilas P, Schmid S, Breitkreuz T, Kallmayer M, Zimmermann A and Eckstein HH: Patient characteristics and in-hospital outcomes of emergency carotid endarterectomy and carotid stenting after stroke in evolution. J Vasc Surg 68: 436-444, 2018.

6. Power GS and Harrison DA: Why try to predict ICU outcomes? Curr Opin Crit Care 20: 544-549, 2014.

7. Verma A and Kole T: International normalized ratio as a predictor of mortality in trauma patients in India. World J Emerg Med 5: 192-195, 2014

8. Leeper CM, Nasr I, McKenna C, Berger RP and Gaines BA: Elevated admission international normalized ratio strongly predicts mortality in victims of abusive head trauma. J Trauma Acute Care Surg 80: 711-716, 2016.

9. Cao C, Martinelli A, Spoelhof B, Llinas RH and Marsh EB: In potential stroke patients on warfarin, the international normalized ratio predicts ischemia. Cerebrovasc Dis Extra 7: 111-119, 2017.

10. Benediktsson S, Frigyesi A and Kander T: Routine coagulation tests on ICU admission are associated with mortality in sepsis: An observational study. Acta Anaesthesiol Scand 61: 790-796, 2017.

11. Johnson AE, Pollard TJ, Shen L, Lehman LW, Feng M, Ghassemi M, Moody B, Szolovits P, Celi LA and Mark RG: MIMIC-III, a freely accessible critical care database. Sci Data 3: 160035, 2016.

12. Johnson A, Stone DJ, Celi LA and Pollard TJ: The MIMIC code repository: Enabling reproducibility in critical care research. J Am Med Inform Assoc 25: 32-39, 2018.

13. Le Gall JR, Lemeshow S and Saulnier F: A new simplified acute physiology score (SAPS II) based on a European/North American multicenter study. Jama 270: 2957-2963, 1993.

14. Steiner C, Elixhauser A and Schnaier J: The healthcare cost and utilization project: An overview. Eff Clin Pract 5: 143-151, 2002.

15. Thompson NR, Fan Y, Dalton JE, Jehi L, Rosenbaum BP, Vadera S and Griffith SD: A new Elixhauser-based comorbidity summary measure to predict in-hospital mortality. Med Care 53: $374-379,2015$
16. Hylek EM, Regan S, Go AS, Hughes RA, Singer DE and Skates SJ: Clinical predictors of prolonged delay in return of the international normalized ratio to within the therapeutic range after excessive anticoagulation with warfarin. Ann Intern Med 135: 393-400, 2001.

17. Makris M, van Veen JJ and Maclean R: Warfarin anticoagulation reversal: Management of the asymptomatic and bleeding patient. J Thromb Thrombolysis 29: 171-181, 2010.

18. Hylek EM and Singer DE: Risk factors for intracranial hemorrhage in outpatients taking warfarin. Ann Intern Med 120 897-902, 1994

19. Hansen P, Zmistowski B, Restrepo C, Parvizi J and Rothman RH: Does international normalized ratio level predict pulmonary embolism? Clin Orthop Relat Res 470: 547-554, 2012.

20. Adike A, Al-Qaisi M, Baffy NJ, Kosiorek H, Pannala R, Aqel B, Faigel DO and Harrison ME: International normalized ratio does not predict gastrointestinal bleeding after endoscopic retrograde cholangiopancreatography in patients with cirrhosis. Gastroenterology Res 10: 177-181, 2017.

21. Cropp JS and Bussey HI: A review of enzyme induction of warfarin metabolism with recommendations for patient management. Pharmacotherapy 17: 917-928, 1997.

22. Rohde LE, de Assis MC and Rabelo ER: Dietary vitamin K intake and anticoagulation in elderly patients. Curr Opin Clin Nutr Metab Care 10: 1-5, 2007.

23. Mlejnsky F, Klein AA, Lindner J, Maruna P, Kvasnicka J, Kvasnicka T, Zima T, Pecha O, Lips M, Rulisek J, et al: A randomised controlled trial of roller versus centrifugal cardiopulmonary bypass pumps in patients undergoing pulmonary endarterectomy. Perfusion 30: 520-528, 2015.

This work is licensed under a Creative Commons Attribution-NonCommercial-NoDerivatives 4.0 International (CC BY-NC-ND 4.0) License. 